\title{
The interaction of descriptive and injunctive social norms in promoting energy conservation
}

\author{
Jacopo Bonan ${ }^{1,3}$, Cristina Cattaneo ${ }^{1}$, Giovanna d'Adda ${ }^{1,2, *}$, and Massimo Tavoni ${ }^{1,3}$ \\ ${ }^{1}$ RFF-CMCC European Institute on Economics and the Environment (EIEE), Centro Euro-Mediterraneo sui \\ Cambiamenti Climatici, Milan, 20144, Italy. \\ ${ }^{2}$ University of Milan, Department of Economics, Management and Quantitative Methods (DEMM), Milan, 20122, \\ Italy. \\ ${ }^{3}$ Politecnico di Milano, Department of Management, Economics and Industrial Engineering, Milan, 20156, Italy. \\ *Correspondence and requests for materials should be addressed to G.D. (email: giovanna.dadda@unimi.it)
}

\section{ABSTRACT}

Behavioural interventions that leverage social norms are widely used to foster energy conservation. For instance, home energy reports combine information on others' behaviour (descriptive feedback) and approval for norm compliant behaviour (injunctive feedback). In a randomized controlled trial, we investigate how descriptive and injunctive feedback interact to affect electricity use, and evaluate the effects of additional normative feedback presented in the form of a descriptive or injunctive energy conservation norm primes. We find that consistent descriptive and injunctive feedback boosts the effectiveness of social information in inducing energy conservation. When descriptive and injunctive feedback are in conflict, conservation behaviour is a function of the relative strength of the two types of feedback. Additional normative feedback produces smaller gains when it reinforces existing information of the same type. These results suggest complementarities between different types of normative messages rather than superiority of any one kind of feedback. 



\section{Introduction}

Social information programmes that provide information on the actions or beliefs of others are widely used interventions to foster behavioural change in several domains ${ }^{1-4}$, including residential resource conservation $^{5-12}$. These programmes typically feature two forms of feedback: descriptive and injunctive. In the case of residential energy, descriptive feedback generally takes the form of information on other households' average energy consumption, while injunctive feedback provides social approval for energy savings. The combination of descriptive and injunctive feedback within the standard design of social information programmes is inspired by the finding that descriptive information alone leads low energy users to increase consumption, and that the addition of injunctive feedback in support of an energy conservation norm prevents this boomerang effect ${ }^{13}$. Injunctive information thus counterbalances descriptive information. However, the experimental evidence on the impact of descriptive and injunctive information when they exert opposing influences on behaviour is mixed and mainly focuses on short-term or selfreported outcomes ${ }^{14,15}$.

Given the wide adoption of communication campaigns relying on social information to promote behavioural change both among policy makers and private firms, it is important to understand how different programme features interact in real world settings ${ }^{16}$. Indeed, impact evaluations of similar programmes find that they are effective in fostering energy savings, but that effect sizes vary widely across contexts and individuals ${ }^{17}$. Prominent explanations for such differential responses rely on heterogeneity of consumers' traits, such as beliefs ${ }^{18}, 19$, misperceptions of one's compliance with the social norm ${ }^{20}$, or personal values ${ }^{21,22}$.

Here we focus on the varying effect of specific features of these messages, and particularly on how the salience, strength and consistency of the feedback they contain differ, and thus affect behavior differently, across users. This could inform the more effective design and targeting of messages and provide more specific and nuanced guidance to prevent similar information campaigns from backfiring ${ }^{16}$. First, we exploit the features of the standard design of home energy reports and isolate the impact of changes in injunctive feedback. Specifically, we examine whether reinforcing the injunctive feedback has different effects on electricity use if it is accompanied by consistent descriptive feedback - as is the case for high energy users, for whom both the injunctive and descriptive information encourage energy conservation - or contrasting descriptive feedback - as is the case for low energy users, for whom conforming with the descriptive feedback entails consumption increases, at odds with the injunctive feedback praising energy saving. Second, we randomize descriptive or injunctive information priming a social norm of energy conservation, and evaluate the effect of strengthening the injunctive feedback in the presence of either the descriptive or the injunctive prime.

We propose a conceptual framework for understanding how different features of social information programmes impact energy conservation that can be articulated in a set of hypotheses, illustrated in Figure 1. First, the effectiveness of a normative message is maximised by the inclusion of consistent feedback of 
different types (i.e., injunctive and descriptive; Figure 1a). Second, when injunctive and descriptive feedback are contrasting (Figure 1b), the strength of each single piece of information matters. The strength of the normative feedback may depend on several factors highlighted in the literature, from the recipient's beliefs on what relevant others think is socially approved of ${ }^{18,23}$ to the degree of consensus or ambiguity around the norm conveyed by the information ${ }^{16,24}$. In our setting, we hypothesize that the effect of the descriptive information increases according to the difference between an individual's electricity consumption and the average consumption of the reference group. The effect of injunctive information instead varies according to the strength of social approval conveyed through visual cues and encouragement messages. Third, additional pieces of consistent feedback of the same type produce smaller savings (Figure 1c).

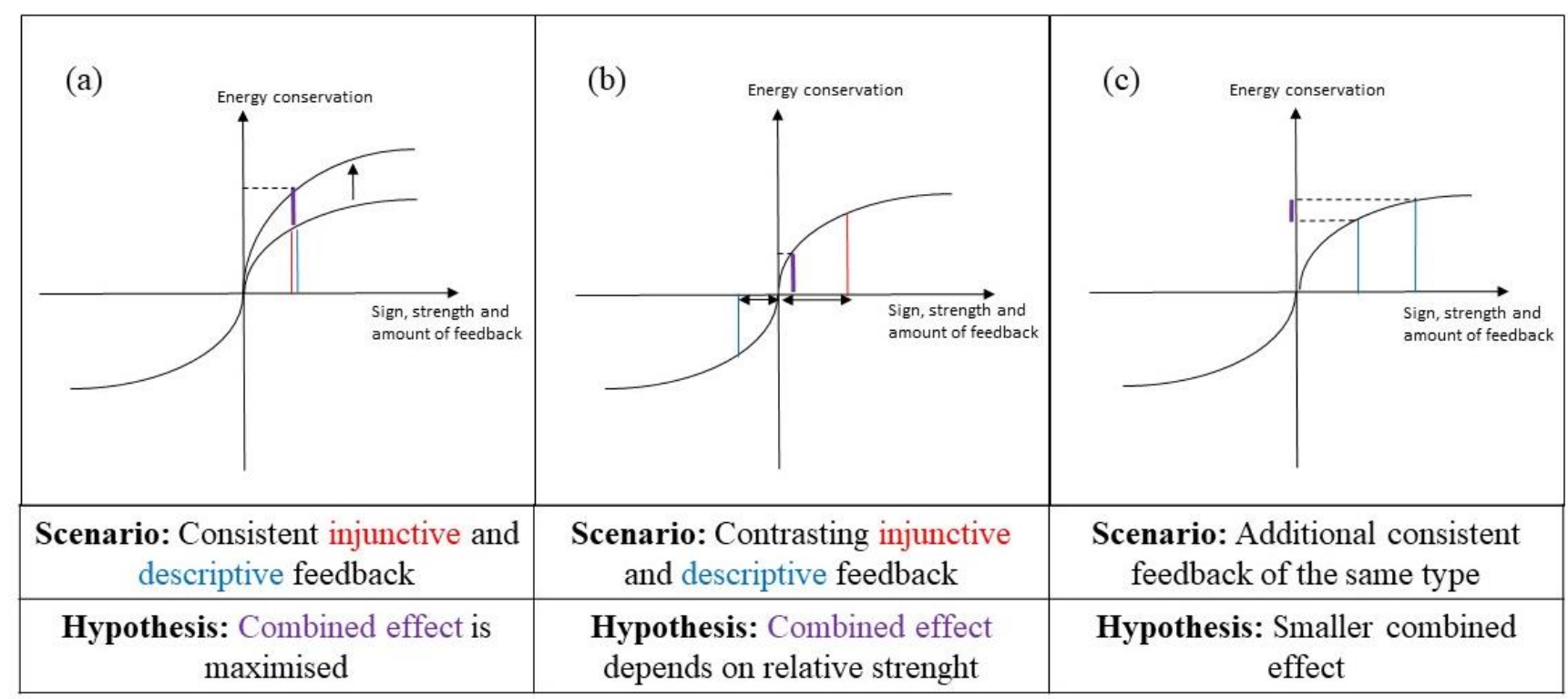

Figure 1. Hypothesized impact of injunctive and descriptive feedback in social information messages. Hypothesized effects of (a) consistent injunctive and descriptive feedback, (b) contrasting injunctive and descriptive feedback, and (c) additional consistent feedback of the same type. The black curve represents the overall impact of the normative message. Injunctive feedback is shown in red, descriptive feedback is shown in blue, and their combined effect is shown in purple. The horizontal axes indicate the sign, strength and amount of normative feedback, where positive feedback values imply messages encouraging electricity savings. The vertical axes represent the behavioural outcome where positive values are associated with behaviour in compliance with the norm, which in our case is energy conservation.

Our results are in line with these hypotheses. First, we find suggestive evidence that the standard social information message induces larger savings among high electricity users who are exposed to consistent descriptive and injunctive feedback compared to low electricity users who are exposed to contrasting descriptive and injunctive feedback. More significantly, reinforcing the injunctive feedback has the largest effect among low electricity users exposed to a consistent descriptive prime. These findings are in line with the notion that injunctive and descriptive feedback have a larger impact on electricity conservation when they pull behaviour in the same rather than in opposite directions. Second, reinforcing 
the injunctive feedback leads to a reduction in consumption, but only among customers with low electricity usage. This shows that the relative strength of the different types of feedback matters when they are contrasting. Such reinforcement has no effect on customers with high consumption. This demonstrates the limited effect of reinforcing one type of normative feedback within a message already containing two consistent pieces of normative information of different types. This is further confirmed by the finding that reinforcing the injunctive feedback has no effect among users exposed to a consistent injunctive prime. Together these findings suggest that additional pieces of feedback have a larger impact when they pull behaviour in the same direction and are of different types. Overall, our results support the presence of synergies between different types of feedback rather than the primacy of any one type of feedback.

\section{Field experiment}

Our setting consists of a randomized controlled trial (RCT) implemented by an Italian energy company providing almost half a million households with information on their electricity use, relative to that of their neighbours ${ }^{6,17}$. The social information is included in a Home Energy Report, distributed to customers via email (eHER). The programme was rolled out in 2016 and involved 464,523 customers (N

$=418,178$ treatment, $\mathrm{N}=46,345$ control). The core feature of the eHER is the neighbour comparison, combining descriptive and injunctive normative information. The descriptive norm graphically compares the customer's electricity use over the previous month to the average use in two reference groups: 100 similar customers living nearby (i.e., neighbours), and the 15 percent most efficient neighbours. The injunctive norm takes the form of thumbs-up symbols next to the descriptive norm graph: 3 thumbs-up ("excellent") for users consuming less than the top 15 percent most efficient neighbours; 2 thumbs-up ("good") for those more efficient than the average neighbour; 1 thumbs-up ("you can do better") for the others. Figure 2 shows the eHER for users receiving 3 and 2 Thumbs-up in Panel (a) and (b), respectively.

We collaborated with the energy company to augment this set-up with a message displayed at the bottom of the eHER delivered in April-May 2018. The utility randomly allocated half of the treated sample at that time $(\mathrm{N}=256,487)$ to receive either the descriptive $(\mathrm{N}=127,899)$ or the injunctive $(\mathrm{N}=128,588)$ message priming an energy conservation norm. The descriptive norm prime (see Figure 2, Supplementary Methods and Supplementary Figure 1) emphasizes that a large majority of customers try to save energy, i.e., adopt behaviours consistent with a social norm of saving electricity. The injunctive norm prime claims that a majority of customers hold electricity saving as a personal value, thus supporting the belief that electricity saving is approved by relevant others. The two primes use fellow customers of the same utility as the reference group. The information on energy saving behaviours and values featured in the primes was taken from an online survey that we conducted with about 3,000 utility customers (see Methods).

We have access to data on monthly electricity consumption from July 2015 to December 2019. The daily average electricity usage, normalized with respect to the control group consumption in the intervention period, is our main outcome variable. Pre-intervention daily electricity usage in a month is 
(a) 3 Thumbs-up

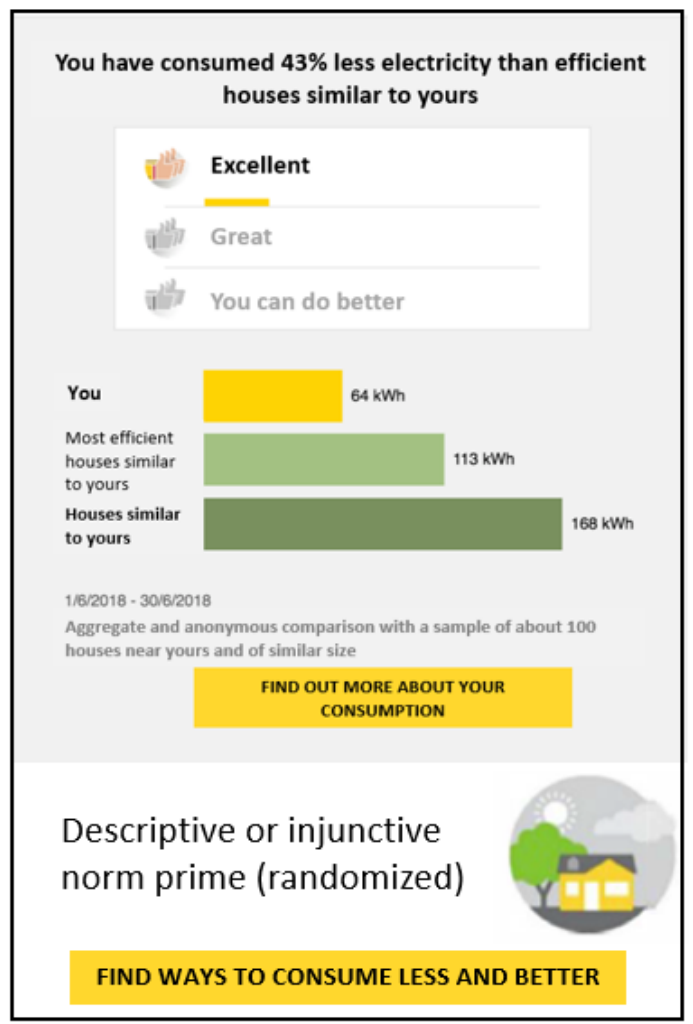

(b) 2 Thumbs-up

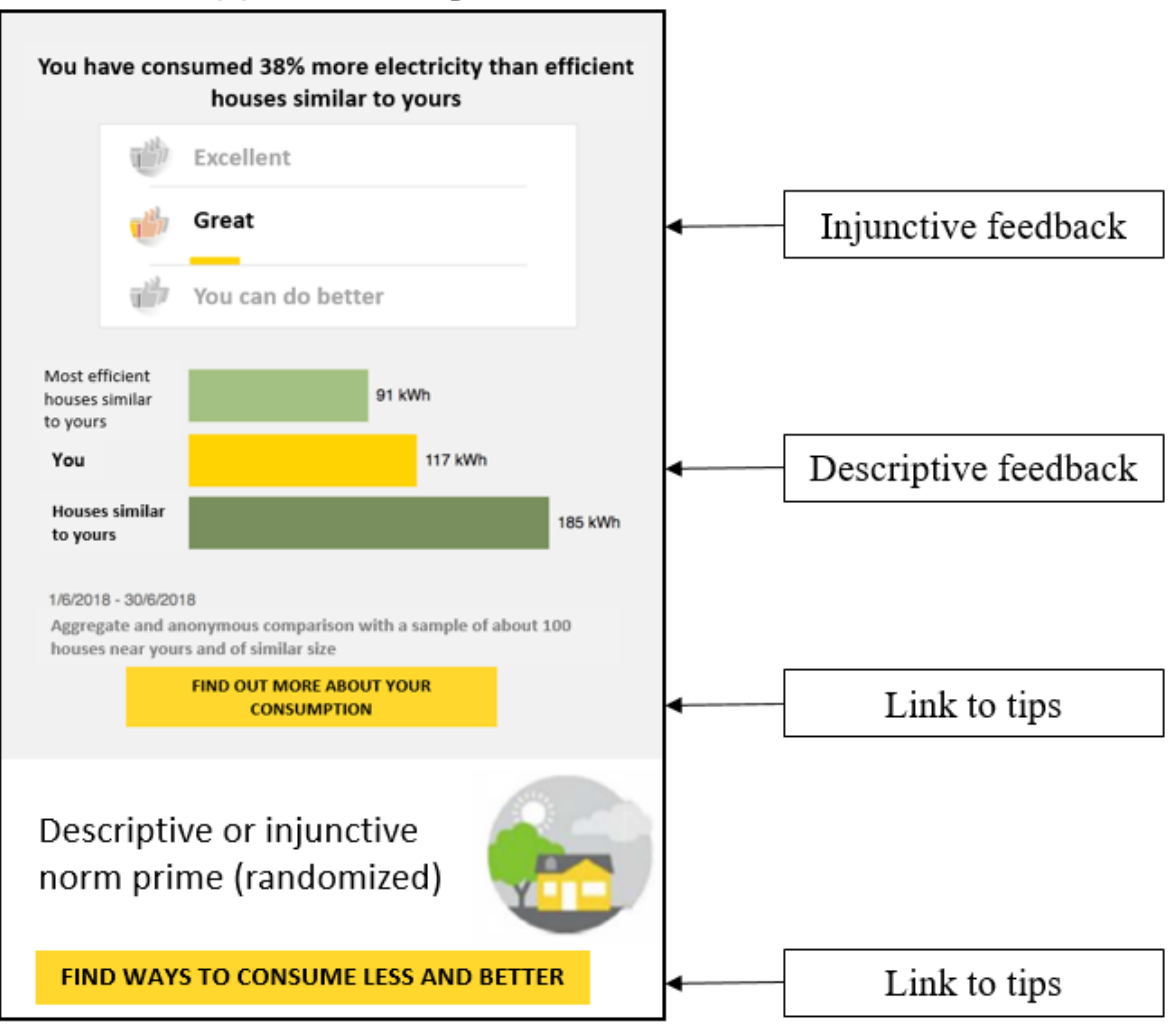

Descriptive prime: Are you reducing energy consumption in your house? More than $80 \%$ of [name of utility] customers take actions in order to save energy.* Even little deeds can have a large impact. Discover our tips to consume less and better.

Injunctive prime: Is saving energy important to you? For more than $80 \%$ of [name of utility] customers saving energy is an important value.* Even little deeds can have a large impact. Discover our tips to consume less and better.

*Survey conducted with a representative sample of [name of utility] customers

Figure 2. Home Energy Report. Layout and content of a Home Energy Report for (a) a user receiving 3 thumbs-up; and (b) a user receiving 2 thumbs-up. Both versions of the report contain the injunctive feedback, i.e., the thumbs-up (top), and the descriptive feedback, i.e., the energy consumption bars (bottom). The figure also displays the position of the randomized norm primes, whose text is reported below the picture of the Home Energy Report.

calculated over the period July 2015-June 2016. Our data also include information on the contents of customers' reports and on whether customers open or click on them. We provide details on the programme implementation and data in the Methods, and descriptive statistics and balance tests in Supplementary Tables 1 and 2 and Supplementary Note 1. Samples are balanced across all available dimensions.

\section{Impact of the social information programme}

The impact evaluation of the standard programme indicates a statistically significant reduction of normalized electricity usage in its first year (coefficient $=-0.353$, s.e. $=0.113, p=0.002$; Equation 1 in 
Methods and Supplementary Table 3, Column 1). The impact of the treatment increases with baseline consumption, although this result is not robust to the measure of electricity consumption used, i.e., discrete or continuous (Supplementary Table 3, Columns 2 and 5); its statistical significance varies with how the sample is defined (Supplementary Table 4) and with the time frame considered (Supplementary Tables 5 - 7); and it does not always hold after multiple hypotheses corrections. Exploiting data on engagement with the reports and on changes in feedback over time, we find that the impact of social information is magnified among users who actually read it and who experience upgrades in feedback (see Supplementary Tables 8 and 9 and Supplementary Note 2 for further details).

While the magnitude of the average savings from the programme $(-0.353 \%)$ is outside of the range of those generated by similar ones in the US $(\min =0.88 \%, \max =2.55 \%)^{18}$, they are in line with the existing evidence from Europe ${ }^{19}$. Various factors, such as lower average consumption in Europe than in the US, the specific features of the programme we studied, or differences in beliefs across contexts may be responsible for these differences. The heterogeneous effects, while not robust and only marginally statistically significant, are qualitatively in line with the existing evidence on the larger impact of social information on high electricity users ${ }^{17,20,25}$ and on the absence of boomerang effects among low users ${ }^{13}$. These results provide initial, albeit weak, support to our conceptual framework. For high users, normative and injunctive feedback pull behaviour in the same direction, resulting in a reduction in electricity almost twice as large as the average treatment effect. For low electricity users, conforming to reference groups' behaviour would motivate a consumption increase ('boomerang'), but the injunctive feedback included in the eHER counterbalances the negative effect of the descriptive feedback. The injunctive feedback therefore induces stronger behavioural reactions among high electricity users, who are also exposed to supporting descriptive feedback, than among low electricity users, for whom the two types of feedback are at odds. While such an interpretation is only suggestive based on the evidence presented so far, it shows how established findings are consistent with our conceptual framework.

\section{Impact of strengthening the injunctive feedback}

Our conceptualization can guide the analysis and interpretation of the effect of intensifying the injunctive feedback, while keeping the descriptive feedback unchanged, for low and high users. We isolate the causal impact of the strength of the injunctive feedback via a regression discontinuity estimation (RD, see Methods). We exploit the fact that the injunctive feedback (number of thumbs-up) changes discretely as a customer's consumption crosses the two thresholds given by the average consumption of the efficient neighbours ( 3 vs. 2 thumbs-up cut-off), and the neighbours' average consumption ( 2 vs. 1 thumbs-up cut$o f f$ ), whereas the descriptive feedback (bars of electricity use) changes imperceptibly across the thresholds. We focus on customers around the thresholds, whose assignment to a given injunctive feedback category is plausibly random. Indeed, while customers belonging to the three normative feedback categories differ on average along various baseline characteristics (see Supplementary Note 3 and Supplementary Table 10), 
individuals close to the thresholds are similar (Supplementary Tables 11 and 12, Columns 1-3).

We conduct two separate RD estimations, one for each cut-off, on the sample of treated customers receiving the eHER sent in April-May $2018(\mathrm{~N}=256,487)$, to allow a direct comparison with the analysis presented below. In each estimation, we compare users in the two feedback categories adjacent to the cut-off and estimate the marginal effect of receiving one additional thumbs-up. Figure 3 presents the results in terms of level changes in electricity usage. While there are no statistically significant changes in the effect of the eHER when crossing the threshold between 1 and 2 thumbs-up (Figure $3 \mathrm{a}$ ), the discrete shift in the injunctive norm reduces electricity use when moving from 2 to 3 thumbs-up (Figure $3 b$ ). The corresponding empirical estimates are presented in Table 1 (Columns 1 and 2).

We can attribute these effects to the social information contained in the report rather than to other content, namely electricity saving tips: tips can only be accessed through a clickable link on the report and we see no difference in click shares across the cut-offs (Supplementary Table 12). The impact of the shift in injunctive feedback is persistent even after 6 and 12 months (see Supplementary Table 13). The results are robust to different specifications of the RD estimate (Table 1).

\section{Figure 3 HERE}

Figure 3. Impact of the injunctive feedback on electricity usage. Each dot represents the average daily electricity usage (in KWh) in the three months following the receipt of the April-May 2018 eHER around the (a) 2 vs. 1 thumbs-up cut off $(n=216,328)$, and (b) 3 vs. 2-thumbs-up cut-off $(n=130,466)$ within evenly-spaced bins. The solid line represents local linear fit, estimated separately on either side of the cut-off with the shaded area showing 95\% confidence intervals. The number of bins is selected through the Integrated Mean Squared Error (IMSE). The running variable (horizontal axis) reports the individual difference between each customer's monthly consumption (in KWh) in the period reported in the eHER and the relevant cut-off. The cut-off is then represented by the vertical line which is set to zero. For positive values of the score, customers get an extra thumbs-up with respect to those with negative values of the score. In Panel (a), customers on the right of the cut-off consume less than the average neighbour and more than the top 15\% most efficient and get 2 thumbs-up. In Panel (b), customers on the right of the cut-off consume less than the $15 \%$ most efficient neighbours and get 3 thumbs-up. Bandwidths

and kernel are set following the data driven process described for formal RD estimations of impacts reported in Table 1.

These findings are consistent with our second hypothesis. Strengthening the approval for electricity savings delivered through the injunctive feedback affects customers around the 3 vs. 2 thumbs-up cutoff more because, for them, it reinforces the relative strength of the injunctive information in the presence of conflicting injunctive and descriptive feedback. On the contrary, and in agreement with our third hypothesis, for consumers around the 2 vs. 1 thumbs-up cut-off the reinforcement of the injunctive feedback has a smaller marginal effect, as it adds strength to already consistent feedback types.

\section{Impact of additional injunctive and descriptive feedback}

To further test the impact of an additional piece of normative information, we combine the features of the 
standard eHER with the randomized addition of descriptive and injunctive information through the primes. To exploit the interaction between the discontinuities in the eHER's injunctive feedback and the randomly delivered primes, we repeat the RD analysis across the two cut-offs ( 2 vs. 1 and 3 vs. 2 thumbs-up) separately for the sub-samples of customers receiving the two types of prime.

Results are reported in Figure 4 and Table 1 (Columns 3 to 6). Across the cut-off between 1 and 2 thumbs-up, we observe no statistically significant changes in consumption, regardless of whether the descriptive (Figure $4 \mathrm{a}$ ) or the injunctive (Figure 4b) prime is present. Conversely, a discrete shift in the injunctive feedback across the 3 vs. 2 cut-off causes electricity reduction, but only when combined with the descriptive prime nudging energy efficiency (Figure 4c). Results are robust to adjustments for multiple hypothesis testing (Table 1) and are persistent over longer time horizons (Supplementary Table 13).

This evidence, consistent with our first hypothesis, suggests synergies between different types of feedback: adding supportive descriptive information increases the impact of the shift in injunctive feedback. The marginal contribution of additional feedback of the same type is instead decreasing: customers exposed to the injunctive prime do not react to the reinforcement of the injunctive feedback across the 3 vs. 2 thumbs-up cut-offs (Figure 4d). Similarly, strengthening the injunctive feedback across the 2 vs. 1 thumbs-up threshold makes no difference, regardless of whether descriptive (Figure 4a) or injunctive information (Figure 4b) is added. In this case, the descriptive and injunctive feedback within the standard neighbour comparison already pull behaviour in the same direction. Further priming either type of normative feedback does not generate incremental electricity conservation.

To determine whether the overall effect of crossing the 3 vs. 2 threshold is exclusively due to the presence within the eHER of the descriptive prime, we performed the RD estimation on a standard eHER (February-March 2018). We found statistically significant effects (coefficient $=-0.855$, s.e. $=0.368, \mathrm{p}=$ 0.02; Table 2). Therefore, the effect of reinforcing the injunctive feedback for low users does not depend on the presence of the descriptive prime within the report. In addition, we observe that the overall effect of crossing the 3 vs. 2 thumbs-up threshold within the standard report is smaller than the same effect when the report is augmented with the descriptive prime (coefficient $=-2.426$, s.e. $=0.619, \mathrm{p}<0.01$; Table 1 , Column 3). This confirms that the combination of consistent descriptive and injunctive information boosts the effectiveness of social information in inducing energy conservation.

The RD estimation provides robust identification of causal effects, which are however only local. We complement it with an estimation of the heterogeneous impact of the primes by the number of thumbs-up

\begin{tabular}{|c|c|c|c|c|c|c|}
\hline & (1) & (2) & (3) & (4) & (5) & (6) \\
\hline & \multicolumn{6}{|c|}{ Electricity usage in the 3 months following the prime } \\
\hline & \multicolumn{2}{|c|}{ All } & \multicolumn{2}{|c|}{ Descriptive prime } & \multicolumn{2}{|c|}{ Injunctive prime } \\
\hline \multicolumn{7}{|l|}{3 vs. 2 Thumbs-up } \\
\hline \multirow[t]{3}{*}{ Conventional } & $-1.217 * * *$ & $-1.165 * *$ & $-2.426 * * *$ & $-2.388 * * *$ & -0.0550 & 0.209 \\
\hline & $(0.461)$ & $(0.456)$ & $(0.619)$ & $(0.611)$ & $(0.686)$ & $(0.676)$ \\
\hline & & & {$[0.001]$} & {$[0.001]$} & [1] & {$[0.66]$} \\
\hline Robust bias-corrected & $-1.130 * *$ & $-1.077 * *$ & $-2.264 * * *$ & $-2.349 * * *$ & -0.0470 & 0.310 \\
\hline
\end{tabular}




$\begin{array}{cccccc}(0.461) & (0.456) & (0.619) & (0.611) & (0.686) & (0.676) \\ & & {[0.002]} & {[0.001]} & {[1]} & {[0.68]}\end{array}$

\begin{tabular}{lcccccc} 
Observations & 130,466 & 130,466 & 65,091 & 65,091 & 65,305 & 65,305 \\
BW select method & one MSE & two MSE & one MSE & two MSE & one MSE & $\begin{array}{c}\text { two MSE } \\
\text { BW above }\end{array}$ \\
BW below & 30.59 & 35.67 & 34.50 & 40.27 & 27.94 & 39.15 \\
BW & 26.95 & 34.50 & 28.89 & 27.94 & 23.14 \\
Effective n. obs above & 31,226 & 36,752 & 17,852 & 21,096 & 10,698 & 20,632 \\
Effective n. obs below & 23,216 & 20,908 & 12,669 & 11,051 & 13,900 & 9,392 \\
& & & & & & \\
2 vs. 1 Thumbs-up & & & & & & \\
Conventional & 0.254 & -0.564 & -0.426 & $-1.046 *$ & 0.491 & -0.161 \\
& $(0.531)$ & $(0.434)$ & $(0.704)$ & $(0.601)$ & $(0.691)$ & $(0.619)$ \\
& & & {$[1]$} & {$[0.14]$} & {$[1]$} & {$[0.66]$} \\
Robust bias-corrected & 0.488 & -0.409 & -0.118 & -0.883 & 0.731 & -0.150 \\
& $(0.531)$ & $(0.434)$ & $(0.704)$ & $(0.601)$ & $(0.691)$ & $(0.619)$ \\
& & & {$[1]$} & {$[0.27]$} & {$[0.771]$} & {$[0.68]$} \\
Observations & 216,328 & 216,328 & 107,729 & 107,729 & 108,477 & 108,477 \\
BW select method & one MSE & two MSE & one MSE & two MSE & one MSE & one MSE \\
BW above & 30.10 & 76.51 & 35.93 & 84.88 & 33.80 & 67.97 \\
BW below & 30.10 & 33.90 & 35.93 & 37.67 & 33.80 & 30.86 \\
Effective n. obs above & 32,148 & 40,038 & 18,765 & 22,115 & 20,015 & 18,306 \\
Effective n. obs below & 35,704 & 69,226 & 21,124 & 36,967 & 17,843 & 31,980 \\
\hline
\end{tabular}

Notes: This table conducts regression discontinuity estimation of average daily energy usage $(\mathrm{KWh})$ in the three months following the receipt of the eHER augmented with the normative prime, normalized by average control group consumption in the Post period, around the 3 vs. 2-thumbs-up and 2 vs. 1-thumbs-up. Estimations in Columns 1 and 2 are on the whole sample, in Columns 3 and 4 are on the sample of customers receiving the descriptive norm prime, in Columns 5 and 6 are on the sample of those receiving the injunctive norm prime. The first row shows the conventional RD estimate, while the second corrects for bias $^{26}$. Bandwidths are selected minimizing the mean squared error (MSE $)^{26,27}$. Odd columns use the same bandwidth on either side of the cut-off, while even columns estimate separate bandwidths. FDR-adjusted q-values in brackets.

$*, * *$, and $* * *$ indicate significance at the $10 \%, 5 \%$, and $1 \%$ levels, respectively. Table 1. RD estimates of the impact of the injunctive norm (number of thumbs-up) on electricity
usage, main and heterogeneous effect by normative prime message.

that customers receive (Equation 3 in Methods). We obtain similar results: while the descriptive prime does not influence consumption on average (coefficient $=0.088$, s.e. $=0.149, \mathrm{p}=0.554$; Table 3 , Column

\section{FIGURE 4 HERE}

Figure 4. Heterogeneous impact of the normative primes at different injunctive feedback cut-offs. Each dot represents the average daily electricity usage (in KWh) in the three months following the receipt of the eHER augmented with the normative prime, within evenly-spaced bins. The solid line represents local linear fit, estimated separately on either side of the cut-off with the shaded area showing $95 \%$ confidence intervals. The number of bins is selected through the Integrated Mean Squared Error (IMSE). The running variable (horizontal axis) reports the individual difference between each customer's monthly consumption (in KWh) in the period reported in the eHER and the relevant cut-off. The running variable (horizontal axis) reports the individual 
difference between each customer's monthly consumption (in KWh) in the period reported in the eHER and the relevant cut-off. For positive values of the score, customers get an extra thumbs-up with respect to those with negative values of the score. a) customers receiving the descriptive norm prime around the 2 vs. 1 Thumbs-up cutoff $(n=107,729)$. b) injunctive norm prime around the 2 vs. 1 Thumbs-up cut-off $(n=108,477)$. c) descriptive norm prime around the 3 vs. 2 Thumbs-up cut-off $(n=65,091)$. $d)$ injunctive norm prime around the 3 vs. 2 Thumbsup cut-off $(\mathrm{n}=65,305)$.

1), it leads to a negative and statistically significant decrease in consumption among customers receiving 3 thumbs-up (coefficient $=-0.959$, s.e. $=0.284, \mathrm{p}<0.001$; Column 4). This negative effect is persistent over six, twelve and 18 months (see Supplementary Note 4 and Supplementary Table 14). Interestingly, the effect of the descriptive prime on the entire group of customers receiving 3 thumbs-up is smaller than the RD estimates for the 3 vs. 2 thumbs-up threshold combined with the descriptive prime. While this simple heterogeneity results should be taken with caution, as thumbs-up are correlated with customers' characteristics (Supplementary Table 10), they can be interpreted in light of our conceptual framework and suggest a potential determinant of the strength of descriptive information. The sample of customers receiving 3 thumbs-up includes the most efficient users, who are far from the 3 vs. 2 thumbs-up threshold. The further a customer is from the threshold, the larger the deviation between her own consumption and the average electricity use, and

\begin{tabular}{lcccc}
\hline & $(1)$ & $(2)$ & $(3)$ & $(4)$ \\
& \multicolumn{4}{c}{ Electricity usage in the 3 months } \\
& following the eHER \\
& 3 vs. 2 Thumbs-up & 2 vs. 1 Thumbs-up \\
\hline Conventional & $-0.855^{* *}$ & $-0.834^{* *}$ & 0.0321 & 0.00172 \\
& $(0.368)$ & $(0.342)$ & $(0.400)$ & $(0.345)$ \\
Robust bias-corrected & $-0.683^{*}$ & $-0.701^{* *}$ & 0.167 & 0.131 \\
& $(0.368)$ & $(0.342)$ & $(0.400)$ & $(0.345)$ \\
& & & & \\
Observations & 134,970 & 134,970 & 224,212 & 224,212 \\
BW select method & one MSE & two MSE & one MSE & two MSE \\
BW above & 25.07 & 35.54 & 26.49 & 77.43 \\
BW below & 25.07 & 21.87 & 26.49 & 24.06 \\
Effective n. obs above & 25,013 & 35,922 & 28,920 & 71,838 \\
Effective n. obs below & 20,674 & 17,915 & 30,963 & 28,136 \\
\hline
\end{tabular}

Notes: This table conducts regression discontinuity estimation of average daily energy usage in the three months following the receipt of the eHER in February-March 2018 (i.e. the one preceding the eHER augmented with the normative prime), normalized by average control group consumption in the Post period, around the 3 vs. 2 Thumbs-up cut-off (Columns 1-2) and 2 vs. 1 Thumbs-up cut-off (Columns 3-4). The first row shows the conventional RD estimate, while the second corrects for bias ${ }^{26}$. Bandwidths are selected minimizing the mean squared error (MSE) ${ }^{26,27}$. Odd columns use the same bandwidth on either side of the cut-off, while even columns estimate separate bandwidths. $*$, **, and $* * *$ indicate significance at the $10 \%, 5 \%$, and $1 \%$ levels, respectively.

Table 2. RD estimates of the impact of the injunctive norm (number of thumbs-up) on electricity usage, February-March 2018 eHER. 
therefore, we argue, the larger the strength of the descriptive norm contained in the standard eHER. In other words, we suggest that in our setting conformity motives become more influential the further away individuals are from the descriptive norm.

This interpretation is confirmed by analysing specifically the effect of the descriptive prime among customers experiencing an upgrade in the injunctive feedback (from 2 to 3 thumbs-up) relative to the previous report. These customers are likely to overlap with the customers included in the RDD estimation, as being close the 3 vs. 2 threshold may result in downgrades and upgrades between reports. The effect of the descriptive prime on these customers is in line with the RD estimates for the 3 vs. 2 thumbs-up threshold combined with the descriptive prime (Figure 5) and larger than the average impact of the descriptive prime on the three thumbs-up sub-sample. Other factors may contribute to these results, but we note that they are consistent with our argument that, when descriptive and normative expectations diverge, the resulting behaviour is a function of the relative strength of the two types of feedback.

\section{Conclusions}

Our findings have implications for the design of social information programmes that rely upon the combination of different types of norms to maximize behavioural change. Similar programmes have

\begin{tabular}{|c|c|c|c|c|}
\hline & \multirow[t]{2}{*}{ (1) } & (2) & \multirow{2}{*}{ (3) } & \multirow{3}{*}{$\begin{array}{c}\text { (4) } \\
3 \text { Thumbs-up } \\
\end{array}$} \\
\hline & & Electricity usage & & \\
\hline & All & 1 Thumbs-up & 2 Thumbs-up & \\
\hline \multirow[t]{2}{*}{ Post } & $-0.300 * * *$ & -0.081 & $-0.459 * * *$ & $-1.190 * * *$ \\
\hline & $(0.113)$ & (0.194) & $(0.128)$ & $(0.213)$ \\
\hline \multirow[t]{3}{*}{ Descr. Prime $*$ Post } & 0.088 & 0.225 & $0.296^{*}$ & $-0.959 * * *$ \\
\hline & $(0.149)$ & $(0.252)$ & $(0.170)$ & $(0.284)$ \\
\hline & & {$[0.142]$} & {$[0.09]$} & {$[0.003]$} \\
\hline \multirow[t]{2}{*}{ Constant } & $102.302 * * *$ & $133.685 * * *$ & $81.618 * * *$ & $50.329 * * *$ \\
\hline & $(0.044)$ & $(0.076)$ & $(0.052)$ & $(0.080)$ \\
\hline Observations & $2,783,190$ & $1,358,951$ & $1,002,787$ & 421,452 \\
\hline R-squared & 0.109 & 0.171 & 0.093 & 0.044 \\
\hline Number of customers & 256,487 & 125,249 & 92,407 & 38,831 \\
\hline
\end{tabular}

Notes: The dependent variable is the average daily electricity usage $(\mathrm{KWh})$, normalized by average control group consumption in the Post period. Reference period for the analysis: Oct 2017- August 2018 (3-months impact). All specifications include customer fixed effects and month by year fixed effects. Standard errors clustered at the customer level in parentheses and FDR-adjusted q-values in brackets; *** $\mathrm{p}<0.01, * * \mathrm{p}<0.05, * \mathrm{p}<0.1$.

Table 3. Impact of the descriptive vs injunctive message on electricity usage, main and heterogeneous effects 
been used in several domains, such as tax compliance ${ }^{28,29}$, charitable giving ${ }^{30}$ or water conservation ${ }^{31}$. According to our conceptual framework and empirical results, no single type of normative information is more effective in absolute terms. Policy makers should instead pay attention to the type of normative feedback they include in their communication; strive to diversify them; avoid conflicting information when it mitigates desirable effects and exploit it otherwise; be aware of the diminishing returns from additional pieces of social information; and of the varying strength of conformity motives acrossindividuals.

Of course, our results may be specific to the context that we study, and particularly to the formulation of injunctive and descriptive feedback characterizing the energy efficiency programme that we evaluate and the normative primes that we designed. For example, the wording and graphical representation of the injunctive feedback in the eHER of this study differ from standard social information programmes widely evaluated $^{6,17,18,21,32}$. Further investigation in other behavioural domains and of alternative formulations of descriptive and injunctive feedback are needed to verify the generalizability of ourfindings.

Finally, while we identify potential determinants of the strength of social information messages, we are far from formulating a comprehensive theoretical model. Such a model should incorporate other important insights from the social information literature, for instance on the role of individual descriptive and normative second-order beliefs, of perceived consensus around the norm, or of misperceptions concerning one's own compliance with the norm. Similarly, within our setting we can only test a few implications of our conceptual framework and cannot control for the influence of other important determinants of the 


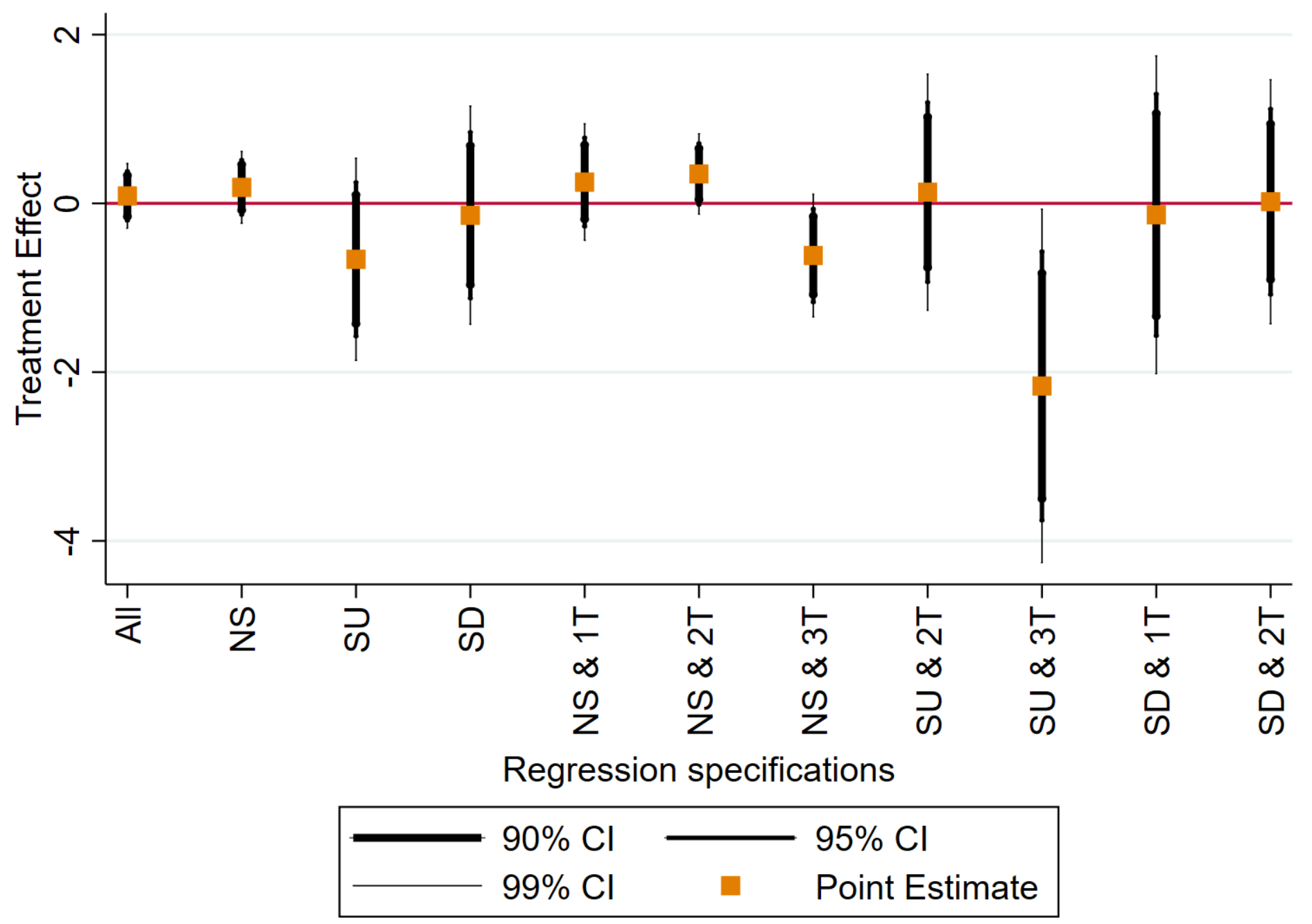

Figure 5. Prime impact by up- and downgrades in injunctive feedback category. The figure plots the coefficient of Descr_Norm * Post on average daily electricity usage (KWh), normalized by average control group consumption in the Post period, estimating equation 3 for the following sub-samples. All: whole sample of customers included in the normative prime trial $(\mathrm{n}=2,783,190)$; NS: customers experiencing no switch in the number of thumbs in the eHER augmented with the normative prime with respect to the previous $(\mathrm{n}=2,334,191)$; SU: switch-up, i.e. improvement in the number of thumbs in the eHER augmented with the normative prime with respect to the previous one ( $\mathrm{n}=239,501)$; SD: switch-down, i.e. decrease in the number of thumbs in the eHER augmented with the normative prime with respect to the previous one $(n=209,498)$; NS 1T: No-switch and 1 Thumbs-up in the eHER augmented with the normative prime ( $\mathrm{n}=1,232,602)$; NS 2T: No-switch and 2 Thumbs-up ( $\mathrm{n}=$ 773,165); NS 3T: No-switch and 3 Thumbs-up $(n=328,424)$; SU 2T: Switch-up and 2 Thumbs-up $(n=146,473)$; SU 3T: Switchup and 3 Thumbs-up ( $(\mathrm{n}=93,028)$; SD 1T: Switch-down and 1 Thumbs-up ( $\mathrm{n}=126,349)$; SD 2T: Switch-down and 2 Thumbsup $(\mathrm{n}=83,149)$.

impact of social information identified in the literature due to lack of data. The predictions from a more comprehensive model should instead be subject to systematic experimental investigation.

\section{Methods}

\section{Programme details}

We evaluate a social information programme designed and implemented by the utility and Opower (acquired by Oracle in 2016). The eHER that constitutes the core feature of the programme differs from 
the standard Opower HER, evaluated in other works $6,17,18,21,32$, under three respects: first, it is delivered by email rather than by post, hence the notation eHER; second, it does not feature a section with energy saving tips: tips can be consulted by interested customers within their personal area on the utility's website, accessible through a clickable link on the eHER; third, the normative feedback is given through thumbs-up accompanied by the expressions "excellent," "good," and "you can do better," rather than through the standard smiley faces coupled with "great," "good," and "above average". The first two differences are consistent with the objective of fostering customers' digital engagement, that the utility primarily wanted to achieve through the programme, while the third is the result of focus groups conducted by the utility and Opower to define the design of theeHER.

Our augmented eHER adds a simple treatment to this basic set-up, in the form of a message at the bottom of the report. We proposed a formulation of the descriptive and injunctive normative messages based on previously collected survey data and collaborated with Opower and the utility to finalise the wording, layout and graphical aspects of the messages. Opower and the utility were responsible for the randomization of the normative messages and the implementation of the test.

In addition to the experimental test discussed in the present study, we manipulated in a similar way the November-December 2017 eHER. The experiment was aimed at testing the impact of environmental identity on energy conservation. It featured a treatment message priming individual environmental selfidentity and a control message encouraging energy conservation ${ }^{22}$. Given that both the NovemberDecember 2017 and the April-May 2018 primes were randomized, participation in the environmental prime test should not affect the results presented here. Nevertheless, we support this claim through further tests reported below.

\section{Sample}

Our sample of analysis is represented by the entire eligible customer base at the time of the start of the programme $(\mathrm{N}=464,523)$. Eligibility criteria were established and verified by the utility and Opower, and include availability of a contact email address and a set of technical requirements, such as living in single family homes, having one year of pre-treatment consumption data without missing, negative or abnormally high usage, and having a sufficient number of neighbours - defined as customers living within a $10 \mathrm{~km}$ radius, similar in terms of type of housing and any other characteristic available to the utility- for the neighbour comparison. The utility is present on the full national territory and the programme was targeted to all eligible customers regardless of their area of residence (Supplementary Figure 2 shows the study sample distribution across Italian municipalities). Moreover, fostering energy conservation was not the main goal of the programme. These considerations alleviate concerns of site selection bias ${ }^{33}$.

Eligible customers were randomly assigned to the treatment $(\mathrm{N}=418,178)$ and control $(\mathrm{N}=46,345)$ groups by the utility through the minmax t-statistic algorithm, depending on baseline consumption and geographic location ${ }^{34}$. The small relative size of the control group was determined by the utility in collaboration with Opower, with the goal of minimising the number of customers not receiving the 
programme, while avoiding issues of statistical power in the evaluation of its impact. The experimental design could eventually capture a Minimum Detectable Effect (MDE with 90\% power and 5\% significance) of about $0.36 \%$. As for heterogeneous effects by pre-treatment usage, MDEs range from 0.8 to $1.2 \%$. These effect sizes are relatively small with respect to the ones found in the literature ${ }^{19,35-37}$.

The programme rolled-out in three waves: July (39\% of treated customers), October (33\%) and December 2016 (28\%). After that, customers received reports bi-monthly. Conditional on being assigned to the treated group, $91 \%$ of customers received at least one eHER, on average 8.8 over the first 24 months of the programme. In April and May 2018, the report augmented with the experimental prime was sent to 256,487 programme participants, randomly assigned to receive the descriptive $(\mathrm{N}=127,899)$ or injunctive $(\mathrm{N}=128,588)$ message. The sample of customers receiving the augmented eHER is smaller than the entire

sample of programme participants at that time $(\mathrm{N}=348,131)$ : since the implementation of the programme was not under the research team's control, we cannot document the reasons for such a discrepancy. We do, however, examine individual correlates of the receipt of the augmented eHER (Supplementary Note 5 and Supplementary Table 15).

About $21.5 \%$ of customers left the dataset between the launch of the programme and August 2018, primarily due to termination of the contract with the utility. We check that attrition is non-differential by treatment status using two-sided t-test in Supplementary Table 1. There, we also report a similar test of whether attrition in the three months following the delivery of the augmented eHER, equal to about 5.8\%, is correlated with the experimental prime treatment. We explore individual correlates of attrition at different points in time using regression analysis in Supplementary Table 16. These allow us to assess the extent to which attrition is a threat to internal and external validity.

\section{Data}

For all customers, we have access to historical electricity consumption data from July 2015 to December 2019. These data are provided by the utility, after being verified by the electricity distributor, and constitute our main outcome variable. Similarly to other works, we compute average daily electricity usage in a month from the total monthly consumption and normalize it by dividing by the average post-period control group consumption and multiplying by $100^{17}$. We also compute the average daily pre-treatment electricity usage as the mean of the average daily consumption in a month between July 2015-June 2016.

We also have access to information on treated customers' engagement with the reports and on the reports' contents. These data are provided by Opower. We know when a eHER was sent and when a customer opened or clicked on a report, although we don't know which one. By opening a report, a customer is able to view the neighbour comparison; by clicking on it, the customer is directed to her personal page on the utility's website, where further information, including energy saving tips and bills, is available. On average, $64 \%$ and $30 \%$ of customers open and click on an eHER, respectively, at least once over the two years from the programme launch. As for the eHER augmented with the normative prime, 55 and $9.7 \%$ of customers opened and clicked at least once in the two months following its receipt, 
respectively.

Reports' content data include customers' relative performance within the reference group and the type of feedback they received within each eHER, in terms of number of thumbs-up. In general, considering all reports in the first 24 months, customers received $20 \%$ of reports with 3 -thumbs-up, $34 \%$ with 2 thumbs-up and $45 \%$ with 1-thumb-up, consistent with the definition of the three feedback categories. Overall, $38 \%$ of customers received the same feedback throughout the period, while the remaining experienced some change. In the majority of cases, customers experienced both upgrades and downgrades. Specifically to the eHER augmented with the randomized prime, 15.1\% of customers received 3-thumbsup, $36.1 \%$ received 2-thumbs-up and 48.8\% 1-thumb-up. As time invariant controls, we use dummies for the main geographical areas, i.e. North-East, North-West, Centre, South and Islands, and the population of the municipality where customers live, obtained by matching the contract municipality data with data on municipalities' characteristics ${ }^{38}$. We miss information on the geographical location of 5,675 customers (about $1.2 \%$ of the sample), equally distributed by treatment status $(p=0.273)$. This reduces the sample size to 459,088 customers whenever the analysis features geographical controls.

Finally, we use data from an online survey conducted on a representative sub-sample of about 3,000 utility customers in April 2017 to inform the design of the norm primes. In particular, questions on actual pro-environmental behaviour -such as turning off the lights when leaving a room and hanging the clothes to dry instead of using dryer- are used to design the descriptive prime, while questions on personal values related to energy conservation - such as whether one feels personally responsible to try to save energy, whether one would act according to her principles if she saved energy, whether one feels morally obliged to save energy-informed the design of the injunctive prime. The survey questions used to elicit personal values and norms were taken from established survey instruments, such as the World Values Survey, and from published studies in environmental psychology 39,40 . More details on the survey can be found in Bonan et al. $(2019)^{22}$.

\section{Balance}

We check that the Opower treatment and control group, and the samples of customers assigned to the descriptive or injunctive prime are balanced across observable characteristics through two-sided t-tests (Supplementary Table 1). In addition, we test for randomization balance across the sub-groups identified by the combination of the April-May 2018 normative prime treatments and the November-December 2017 environmental prime treatments, mentioned above (Supplementary Table 2). This is done through an F-test of joint significance of sub-treatment dummies regressed on the observable characteristics. We confirm that balance generally holds.

\section{Impact of the programme}

We evaluate the impact of the programme by estimating the following model: 


$$
y_{i t}=B_{1} \text { Post }_{i t}+B_{2} \text { Program }_{i} * \text { Post }_{i t}+h_{t}+g_{i}+\varepsilon_{i t}
$$

where $y_{i t}$ is customer's $i$ normalized average daily consumption in month $t$. Program is a treatment indicator, Post is a dummy variable which becomes one when customers receive the first eHER. Given the staggered phase-in of the programme, to allow the identification through difference-in-differences, we randomly assign control customers to the three programme start waves, with the same proportions as treated customers. This implies that Post becomes one at the beginning of each wave for the same share of treated and control customers. The regression also includes month-by-year fixed effects, $h_{t}$, and household fixed effects $g_{i}$. Standard errors are clustered at the level of household, to allow for the presence of within-customer correlation over time in the error term ${ }^{41}$. The ATEs can be interpreted as percentage change (Supplementary Table 3).

We examine the differential response to the eHER depending on pre-treatment electricity use by interacting it with the Program * Post dummy. We express electricity usage with a continuous variable and with dummies for quartiles. To allow for differential post-treatment trends, we also interact the Post dummy with pre-treatment usage. To adjust for multiple hypothesis testing, in the sub-group analysis we compute the sharpened two-stage q-values (FDR-adjusted q-values) ${ }^{42}$. We analyse ex post power by calculating Minimum Detectable Effects (MDE) with $90 \%$ power and $5 \%$ significance $^{43}$. As a robustness check, we estimate the main and heterogeneous effects of the programme on the sub-sample of customers for whom information on geographical location is available (Supplementary Table 4).

We evaluate the main and heterogeneous impact of social information over the first and the first two years of the programme. In further analysis, we extend the treatment period until December 2019 and explore in greater detail the persistence of treatment effects (Supplementary Tables 5 - 7).

As in other works ${ }^{6,17}$, our treatment effects are Intent-To-Treat estimates, computed on the full sample of eligible customers, regardless of whether they opted out of the programme or did not open the reports. We keep customers who do not receive or read reports in the analysis to maintain the balance between treatment and control group and avoid selection issues from affecting our results. By doing this, we are likely to underestimate the effect of the programme on the group of customers initially assigned to receive the eHER and who actually see the treatment communication. In additional analysis, we examine the role of engagement with the programme on treatment impacts, by instrumenting opening the eHER and clicking on it with treatment status, and reporting the Local Average treatment Effect (LATE) (Supplementary Table 8). We do this in cross-section. The outcome variable is the normalized average electricity usage in the 24 months following the launch of the programme. The specifications also include pre-treatment electricity usage.

We further exploit our data on the content of the reports, specifically on the number of thumbs-up received within each eHER, to examine heterogeneous effects of the treatment depending on whether customers are upgraded or downgraded with respect to the previous report. We do it by restricting the analysis to the months when the eHERs are sent and by focusing on normalized daily electricity usage in 
the three months following each report. Specifications include customer and month by year fixed effects (Supplementary Table 9).

\section{Regression Discontinuity Estimation}

We use RD to estimate the impact of changes in the injunctive feedback included in the neighbour comparison. We justify this approach by showing that customers receiving one, two or three thumbs-up within the April-May 2018 eHER are different under many respects, that may also correlate with the impact of the treatment (Supplementary Table 10). The RD approach allows us to eliminate the influence of confounding factors on the estimated effect of changing feedback category, by focusing on customers for whom the number of thumbs-up is as random. The price we pay for the improved identification of effects is that the impacts estimated through RD are local, specific to a neighbourhood of the thresholds.

In our RD framework, the running variables, $X_{i 1}$ and $X_{i 2}$, are customer's $i$ monthly electricity usage (in KWh). The cut-offs, $c_{i 1}$ and $c_{i 2}$, are the electricity usage of the fifteenth percentile and the overall average electricity usage among the neighbours, respectively. . The assignment variable $T_{i 1}$ takes the value of one when the customer's usage lies above $c_{i 1}\left(X_{i 1}>c_{i 1}\right)$ and zero otherwise. Similarly, $T_{21}$ takes the value of one when the customer's usage lies between $c_{i 1}$ and $c_{i 2}$ and zero otherwise. We estimate the following equations:

$$
\begin{aligned}
& Y_{i}=\lambda_{0}^{1}+\eta^{1} T_{i 1}+f\left(X_{i 1}-c_{i 1}\right)+\varepsilon_{1}^{1} \\
& Y_{i}=\lambda_{0}^{2}+\eta^{2} T_{i 2}+f\left(X_{i 2}-c_{i 2}\right)+\varepsilon_{1}^{2}
\end{aligned}
$$

where $Y_{i}$ is customer's $i$ normalized average daily electricity usage in the three months following the receipt of the report containing the normative prime. Model $2 \mathrm{a}$ is estimated on customers who received either three or two thumbs-up (e.g. with $X_{i 1}>c_{i 1}$ or $c_{i 1}<X_{i 1}<c_{i 2}$ ), while model $2 \mathrm{~b}$ includes those receiving either two or one thumb-up (e.g $X_{i 1}<c_{i 1}$ ). Our main specification uses a non-parametric approach (local polynomial point estimation), which amounts to fitting two linear regressions on customers respectively close to the left and to the right of the cut-off ${ }^{44}, 45$. This approach uses only observations within a bandwidth from the cut-off. The selection of the bandwidth is data driven and aims at minimizing the mean squared error (MSE) of the local polynomial RD estimator. We compute the point estimates using both one common bandwidth on both sides of the cut-off and two distinct bandwidths. We use linear polynomial for $f($ ) which represents a good trade-off between simplicity, precision and stability ${ }^{46}$. Observations within the bandwidth are weighted through a triangular kernel function, which assigns zero weight to observations outside the bandwidth and positive weights within it, decreasing symmetrically and linearly as the distance from the cut-off increases. The triangular kernel has desirable asymptotic optimality properties ${ }^{46}$. We show point estimates under conventional (from parametric least-squares estimation) and bias-corrected robust standard errors, which deliver valid inference under MSE-optimal bandwidth selection ${ }^{45}$ (Table 1). 
Identification within RD design relies on the assumption that customers have no full control over the running variable and hence are unable to manipulate their position in the distribution of electricity usage within their reference group. Manipulation should not be a threat to our identification for several reasons. First, customers do not know the algorithm for the selection of the reference group and assignment of thumbs-up. Second, while people may be able to control their own electricity usage, they have no control on their neighbours' consumption, which determines the unpredictability of the consumption level associated to the different cut-offs every round. Third, people do not gain any direct benefit from an extra thumbs-up, apart from individual satisfaction, hence the incentives to game the system should be minimal. We test this identification assumption by checking for discontinuities in the density of the running variable at the thresholds, using a non-parametric density estimator based on local polynomial techniques ${ }^{47}$. We do the same with pre-treatment covariates, following the procedure suggested by the literature ${ }^{48}$ (Supplementary Table 11).

We also perform the RD estimation on the probability that customers open or click on the April-May 2018 eHER. This analysis is aimed at supporting the claim that the effects we find on consumption are due to the neighbour comparison, rather than to discontinuous changes in unobserved determinants of open rates or in the likelihood of viewing electricity saving tips at the thresholds (Supplementary Table 12).

We repeat the test of the RD identifying assumption and the RD estimation using a standard eHER, delivered in February-March 2018, to rule out that our results are due to the presence of the normative primes (Supplementary Note 6, Supplementary Tables 17 and 2) and RD effects over the following six and twelve months (Supplementary Table 13).

\section{Impact of the normative primes}

We repeat the RD estimation separately on the sub-samples of customers randomly allocated to the descriptive and injunctive primes to obtain local marginal effects of moving across injunctive feedback categories by prime treatment. We adjust for multiple comparisons ${ }^{42}$.

We also assess the impact of receiving the eHER augmented by the descriptive vs injunctive prime, by estimating the following model:

$$
y_{i t}=\alpha_{1} \text { Post }_{i t}+\alpha_{2} \text { Descr_Norm } i * \text { Post }_{i t}+h_{t}+g_{i}+_{i t}
$$

where $y_{i t}$ is customer's $i$ normalized average daily consumption over the billing period in month $t$. Descr_Norm takes the value of one for the descriptive prime and zero for the injunctive prime. Post

becomes one when customers receive the eHER containing the prime (roughly half in April and half in May 2018). As before, $h_{t}$ represents month-by-year fixed effects and $g_{i}$ are household fixed effects. Standard errors are clustered at the household level (Table 3). The regression is run on the sample of treated customers at that time $(\mathrm{N}=256,487)$ and considers the period spanning from October 2017 to August 2018, but we also explore the persistence of the effects until December 2019, i.e., 18 months after 
the prime (Supplementary Table 14). We also estimate equation 3 for the sub-samples of customers receiving one, two or three thumbs-up, overall and separately for customers upgraded or downgraded with respect to the previous report, to examine the heterogeneous impact of the augmented eHER.

We test that these results are not due to interaction effects with the November-December 2017 environmental identity test, by adding to equation 3 an interaction term between Post, Descr_Norm and a dummy equal to 1 if a customer was assigned to the environmental prime treatment (Supplementary Table 18).

\section{Pre-registration}

We analyse the impact of the standard and augmented eHER using non-experimental, RD techniques. In terms of individual level data, our analysis relies only on customers' consumption, location and content of the eHER. For these reasons, the analysis we present was not pre-specified in a Pre-Analysis Plan (PAP): detailed pre-specification is warranted when, within the impact evaluation of field experiments, sub-group analysis is expected to be important and there is possibility to cherry-pick the dimensions of heterogeneity to focus on, or a party to the study has a vested interest ${ }^{49}$. These conditions do not apply to our case.

\section{Data Availability Statement}

The data that support the findings of this study are proprietary data of the energy company and cannot be shared publicly. To inquire about access to the proprietary data, please get in touch with M.T. (massimo.tavoni@polimi.it).

\section{Code Availability Statement}

The replication code is available on Open Science Framework at https://osf.io/wz8gb/.

\section{Ethics Declarations}

The authors declare no competing interests. Ethical approval for the use of the data that support the findings of this study was granted by the Institutional Review Board at Politecnico di Milano (approval number 04/2017). Consent for their administrative data to be used for research was given by users as part of the privacy consent statements that they signed with the utility.

\section{Acknowledgements}

The research leading to these results received funding from the European Research Council under the European Unions Seventh Framework Program (FP7/2007-2013) / ERC grant agreement no. 336155—project COBHAM 'The role of consumer behavior and heterogeneity in the integrated assessment of energy and climate policies'.

\section{Competing interests}

The authors declare no competing interests. 


\section{References}

1. Frey, B. S. \& Meier, S. Social Comparisons and Pro-social Behavior: Testing "Conditional Cooperation" in a Field Experiment. Am. Econ. Rev. 94, 1717-1722, DOI: 10.1257/0002828043052187 (2004).

2. Shang, J. \& Croson, R. A Field Experiment in Charitable Contribution: The Impact of Social Information on the Voluntary Provision of Public Goods. The Econ. J. 119, 1422-1439, DOI: 10.1111/j.1468-0297.2009.02267.x (2009). 00291.

3. Gerber, A. S. \& Rogers, T. Descriptive social norms and motivation to vote: Everybody's voting and so should you. The J. Polit. 71, 178-191, DOI: 10.1017/S0022381608090117 (2009). https: //doi.org/10.1017/S0022381608090117.

4. Beshears, J., Choi, J. J., Laibson, D., Madrian, B. C. \& Milkman, K. L. The Effect of Providing Peer Information on Retirement Savings Decisions. The J. Finance 70, 1161-1201, DOI: 10.1111/jofi. 12258 (2015).

5. Allcott, H., Mullainathan, S. \& Taubinsky, D. Externalizing the Internality. New York Univ. Work. Pap. (2011). 00008.

6. Allcott, H. \& Rogers, T. The Short-Run and Long-Run Effects of Behavioral Interventions: Experimental Evidence from Energy Conservation. Am. Econ. Rev. 104, 3003-3037, DOI: 10.1257/aer.104.10.3003 (2014).

7. Ayres, I., Raseman, S. \& Shih, A. Evidence from two large field experiments that peer comparison feedback can reduce residential energy usage. The J. Law, Econ. Organ. 29, 992-1022, DOI: 10.1093/jleo/ews020 (2013). /oup/backfile/content_public/journal/jleo/29/5/10.1093/jleo/ews020/2/ ews020.pdf.

8. Brent, D. A., Cook, J. H. \& Olsen, S. Social comparisons, household water use, and participation in utility conservation programs: Evidence from three randomized trials. J. Assoc. Environ. Resour. Econ. 2, 597-627, DOI: 10.1086/683427 (2015). https://doi.org/10.1086/683427.

9. Ferraro, P. J. \& Price, M. K. Using nonpecuniary strategies to influence behavior: evidence from a large-scale field experiment. Rev. Econ. Stat. 95, 64-73 (2013). 00052.

10. Ferraro, P. J., Miranda, J. J. \& Price, M. K. The persistence of treatment effects with norm-based policy instruments: Evidence from a randomized environmental policy experiment. Am. Econ. Rev. 101, 318-22, DOI: 10.1257/aer.101.3.318 (2011).

11. Ferraro, P. J. \& Miranda, J. J. Heterogeneous treatment effects and mechanisms in information-based environmental policies: Evidence from a large-scale field experiment. Resour. Energy Econ. 35, 356379, DOI: https://doi.org/10.1016/j.reseneeco.2013.04.001 (2013). 
12. Jaime Torres, M. M. \& Carlsson, F. Direct and spillover effects of a social information campaign on residential water-savings. J. Environ. Econ. Manag. 92, 222-243, DOI:10.1016/j.jeem.2018.08.005 (2018).

13. Schultz, P. W., Nolan, J. M., Cialdini, R. B., Goldstein, N. J. \& Griskevicius, V. The constructive, destructive, and reconstructive power of social norms. Psychol. Sci. 18, 429-434 (2007). 00749.

14. Bicchieri, C. \& Xiao, E. Do the right thing: but only if others do so. J. Behav. Decis. Mak. 22, 191208, DOI: 10.1002/bdm.621 (2009). 00137.

15. Meisel, M. K. \& Goodie, A. S. Descriptive and injunctive social norms' interactive role in gambling behavior. Psychol. Addict. Behav. 28, 592-598, DOI: 10.1037/a0036444 (2014). Place: US Publisher: American Psychological Association.

16. Bicchieri, C. \& Dimant, E. Nudging with care: the risks and benefits of social information. Public Choice. In Press, DOI: 10.1007/s11127-019-00684-6 (2019).

17. Allcott, H. Social norms and energy conservation. J. Public Econ. 95, 1082-1095, DOI: 10.1016/j. jpubeco.2011.03.003 (2011). 00344.

18. Jachimowicz, J. M., Hauser, O. P., O’Brien, J. D., Sherman, E. \& Galinsky, A. D. The critical role of second-order normative beliefs in predicting energy conservation. Nat. Hum. Behav. 2, 757-764, DOI: 10.1038/s41562-018-0434-0 (2018). Number: 10 Publisher: Nature Publishing Group.

19. Andor, M. A., Gerster, A., Peters, J. \& Schmidt, C. M. Social norms and energy conservation beyond the US. J. Environ. Econ. Manag. 102351, DOI: 10.1016/j.jeem.2020.102351 (2020).

20. Byrne, D. P., Nauze, A. L. \& Martin, L. A. Tell me something i don't already know: Informedness and the impact of information programs. The Rev.Econ. Stat. 100, 510-527, DOI: 10.1162/rest_a_00695 (2018). https://doi.org/10.1162/rest_a_00695.

21. Costa, D. L. \& Kahn, M. E. Energy conservation "nudges" and environmentalist ideology: evidence from a randomized residential electricity field experiment. J. Eur. Econ. Assoc. 11, 680-702(2013). 00191.

22. Bonan, J., Cattaneo, C., D’Adda, G. \& Tavoni, M. Can we make social information programs more effective? The role of identity and values. RFF-CMCC Working Paper 19-21, (2019).

23. Bursztyn, L., González, A. L. \& Yanagizawa-Drott, D. Misperceived Social Norms: Female Labor Force Participation in Saudi Arabia. Working Paper 24736, National Bureau of Economic Research (2018). DOI: 10.3386/w24736. Series: Working Paper Series.

24. D’Adda, G., Dufwenberg, M., Passarelli, F. \& Tabellini, G. Partial Norms. SSRN Scholarly Paper ID 3362021, Social Science Research Network, Rochester, NY (2019).

25. List, J. A., Metcalfe, R. D., Price, M. K. \& Rundhammer, F. Harnessing Policy Complementarities to Conserve Energy: Evidence from a Natural Field Experiment. Working Paper 23355, National 
Bureau of Economic Research (2017). DOI: 10.3386/w23355. 00000.

26. Calonico, S., Cattaneo, M. D. \& Titiunik, R. Robust Nonparametric Confidence Intervals for Regression-Discontinuity Designs. Econometrica 82, 2295-2326, DOI: 10.3982/ECTA11757 (2014).

27. Calonico, S., Cattaneo, M. D., Farrell, M. H. \& Titiunik, R. Regression Discontinuity Designs Using Covariates. The Rev. Econ. Stat. 101, 442-451 (2019).

28. Hallsworth, M., List, J. A., Metcalfe, R. D. \& Vlaev, I. The behavioralist as tax collector: Using natural field experiments to enhance tax compliance. J. Public Econ. 148, 14-31, DOI: 10.1016/j. jpubeco.2017.02.003 (2017).

29. Bobek, D. D., Hageman, A. M. \& Kelliher, C. F. Analyzing the Role of Social Norms in Tax Compliance Behavior. J. Bus. Ethics 115, 451-468, DOI: 10.1007/s10551-012-1390-7 (2013).

30. Krupka, E. L. \& Croson, R. T. A. The differential impact of social norms cues on charitable contributions. J. Econ. Behav. \& Organ. 128, 149-158, DOI: 10.1016/j.jebo.2016.05.005 (2016).

31. Bhanot, S. Isolating The Effect Of Injunctive Norms On Conservation Behavior: New Evidence From A Field Experiment In California. Organ. Behav. And Hum. Decis. Process. In Press, DOI: 10.1016/j.obhdp. 2018.11 .002 (2018).

32. Allcott, H. \& Taubinsky, D. Evaluating Behaviorally Motivated Policy: Experimental Evidence from the Lightbulb Market. Am. Econ. Rev. 105, 2501-38, DOI: 10.1257/aer.20131564 (2015). 00040.

33. Allcott, H. Site Selection Bias in Program Evaluation *. The Q. J. Econ. 130, 1117-1165, DOI: 10.1093/qje/qjv015 (2015).

34. Bruhn, M. \& McKenzie, D. In pursuit of balance: Randomization in practice in development field experiments. Am. Econ. Journal: Appl. Econ. 1, 200-232, DOI: 10.1257/app.1.4.200 (2009).

35. Andor, M. A. \& Fels, K. M. Behavioral economics and energy conservation - a systematic review of non-price interventions and their causal effects. Ecol. Econ. 148, 178 - 210, DOI: https://doi.org/10. 1016/j.ecolecon.2018.01.018 (2018).

36. Farrow, K., Grolleau, G. \& Ibanez, L. Social norms and pro-environmental behavior: A review of the evidence. Ecol. Econ. 140, 1 - 13, DOI: https://doi.org/10.1016/j.ecolecon.2017.04.017 (2017).

37. Delmas, M. A., Fischlein, M. \& Asensio, O. I. Information strategies and energy conservation behavior: A meta-analysis of experimental studies from 1975 to 2012. Energy Policy 61, 729-739 (2013). 00014.

38. Guiso, L., Sapienza, P.\& Zingales, L. Long-Term Persistence. J. Eur. Econ. Assoc. 14, 1401-1436, DOI: 10.1111/jeea.12177 (2016). Publisher: Oxford Academic.

39. Steg, L., Perlaviciute, G., van der Werff, E. \& Lurvink, J. The significance of hedonic values for environmentally relevant attitudes, preferences, and actions. Environ. Behav. 46, 163-192, DOI: 
10.1177/0013916512454730 (2014).

40. van der Werff, E., Steg, L. \& Keizer, K. The value of environmental self-identity: The relationship between biospheric values, environmental self-identity and environmental preferences, intentions and behaviour. J. Environ. Psychol. 34, 55-63, DOI: 10.1016/j.jenvp.2012.12.006 (2013).

41. Bertrand, M., Duflo, E. \& Mullainathan, S. How Much Should We Trust Differences-In-Differences Estimates? The Q. J. Econ. 119, 249-275, DOI: 10.1162/003355304772839588(2004).

42. Benjamini, Y., Krieger, A. M. \& Yekutieli, D. Adaptive linear step-up procedures that control the false discovery rate. Biometrika 93, 491-507, DOI: 10.1093/biomet/93.3.491 (2006).

43. Haushofer, J. \& Shapiro, J. The Short-term Impact of Unconditional Cash Transfers to the Poor: Experimental Evidence from Kenya. The Q. J. Econ. 131, 1973-2042, DOI: 10.1093/qje/qjw025 (2016).

44. Imbens, G. W. \& Lemieux, T. Regression discontinuity designs: A guide to practice. J. Econom. 142, 615 -635, DOI: https://doi.org/10.1016/j.jeconom.2007.05.001 (2008). The regression discontinuity design: Theory and applications.

45. Calonico, S., Cattaneo, M. D. \& Titiunik, R. Robust nonparametric confidence intervals for regressiondiscontinuity designs. Econometrica 82, 2295-2326, DOI: 10.3982/ECTA11757 (2014). https: //onlinelibrary.wiley.com/doi/pdf/10.3982/ECTA11757.

46. Cattaneo, M. D., Idrobo, N. \& Titiunik, R. A Practical Introduction to Regression Discontinuity Designs: Foundations. Elements in Quantitative and Computational Methods for the Social Sciences (Cambridge University Press, 2019).

47. Cattaneo, M. D., Jansson, M. \& Ma, X. Simple local polynomial density estimators. J. Am. Stat. Assoc. 0,1-7,DOI:10.1080/01621459.2019.1635480(2019). https://doi.org/10.1080/01621459.2019. 1635480 .

48. Canay, I. A. \& Kamat, V. Approximate Permutation Tests and Induced Order Statistics in the Regression Discontinuity Design. The Rev. Econ. Stud. 85, 1577-1608, DOI: 10.1093/restud/rdx062 (2018).

49. Banerjee, A. et al. In Praise of Moderation: Suggestions for the Scope and Use of Pre-Analysis Plans for RCTs in Economics. Natl. Bureau Econ. Res. Work. Pap. Ser. (2020). Institution: National Bureau of Economic Research Number: w26993.

50. Cattaneo, M. D., Jansson, M. \& Ma, X. Manipulation testing based on density discontinuity. The Stata J. 18, 234-261, DOI: 10.1177/1536867X1801800115 (2018). https://doi.org/10.1177/ $1536867 X 1801800115$.

51. Lee, D. S. \& Lemieux, T. Regression discontinuity designs in economics. J. Econ. Lit. 48, 281-355, DOI: $10.1257 /$ jel.48.2.281 (2010). 